\title{
Natural metrics and composition operators in generalized hyperbolic function spaces
}

\section{A El-Sayed Ahmed ${ }^{*}$}

\section{"Correspondence:}

ahsayed80@hotmail.com

Faculty of Science, Mathematics

Department, Sohag University,

Sohag, 82524, Egypt

Current address: Faculty of Science,

Mathematics Department, Taif

University, Box 888, El-Hawiah, Taif,

Saudi Arabia

\begin{abstract}
In this paper, we define some generalized hyperbolic function classes. We also introduce natural metrics in the generalized hyperbolic $(p, \alpha)$-Bloch and in the generalized hyperbolic $Q^{*}(p, s)$ classes. These classes are shown to be complete metric spaces with respect to the corresponding metrics. Moreover, boundedness and compactness the composition operators $C_{\phi}$ acting from the generalized hyperbolic $(p, \alpha)$-Bloch class to the class $Q^{*}(p, s)$ are characterized by conditions depending on an analytic self-map $\phi: \mathbb{D} \rightarrow \mathbb{D}$.
\end{abstract}

MSC: 47B38; 46E15

Keywords: hyperbolic classes; composition operators; $(p, \alpha)$-Bloch space; $Q^{*}(p, s)$ classes

\section{Introduction}

Let $\mathbb{D}=\{z:|z|<1\}$ be the open unit disc of the complex plane $\mathbb{C}, \partial \mathbb{D}$ its boundary. Let $\mathcal{H}(\mathbb{D})$ denote the space of all analytic functions in $\mathbb{D}$ and let $B(\mathbb{D})$ be the subset of $\mathcal{H}(\mathbb{D})$ consisting of those $f \in \mathcal{H}(\mathbb{D})$ for which $|f(z)|<1$ for all $z \in \mathbb{D}$. Also, $d A(z)$ be the normalized area measure on $\mathbb{D}$ so that $A(\mathbb{D}) \equiv 1$. The usual $\alpha$-Bloch spaces $\mathcal{B}_{\alpha}$ and $\mathcal{B}_{\alpha, 0}$ are defined as the sets of those $f \in \mathcal{H}(\mathbb{D})$ for which

$$
\|f\|_{\mathcal{B}_{\alpha}}=\sup _{z \in \mathbb{D}}\left|f^{\prime}(z)\right|\left(1-|z|^{2}\right)^{\alpha}<\infty
$$

and

$$
\lim _{|z| \rightarrow 1}\left|f^{\prime}(z)\right|\left(1-|z|^{2}\right)^{\alpha}=0
$$

respectively. Now, we will give the following definition:

Definition 1.1 The $(p, \alpha)$-Bloch spaces $\mathcal{B}_{p, \alpha}$ and $\mathcal{B}_{p, \alpha, 0}$ are defined as the sets of those $f \in \mathcal{H}(\mathbb{D})$ for which

$$
\|f\|_{\mathcal{B}_{p, \alpha}}=\frac{p}{2} \sup _{z \in \mathbb{D}}|f(z)|^{\frac{p}{2}-1}\left|f^{\prime}(z)\right|\left(1-|z|^{2}\right)^{\alpha}<\infty,
$$

and

$$
\lim _{|z| \rightarrow 1}|f(z)|^{\frac{p}{2}-1}\left|f^{\prime}(z)\right|\left(1-|z|^{2}\right)^{\alpha}=0,
$$

where $0<p, \alpha<\infty$.

\section{Springer}

( 2012 El-Sayed Ahmed; licensee Springer. This is an Open Access article distributed under the terms of the Creative Commons Attribution License (http://creativecommons.org/licenses/by/2.0), which permits unrestricted use, distribution, and reproduction in any medium, provided the original work is properly cited. 
Remark 1.1 The definition of $(p, \alpha)$-Bloch spaces is introduced in the present paper for the first time. One should note that, if we put $p=2$ in Definition 1.1, we will obtain the spaces $\mathcal{B}_{\alpha}$ and $\mathcal{B}_{\alpha, 0}$.

Remark 1.2 $(p, \alpha)$-Bloch space is very useful in some calculations in this paper and it can be also used to study some other operators like integral operators (see [12]).

If $(X, d)$ is a metric space, we denote the open and closed balls with center $x$ and radius $r>$ 0 by $B(x, r):=\{y \in X: d(y, x)<r\}$ and $\bar{B}(x, r):=\{y \in X: d(y, x)=r\}$, respectively. The wellknown hyperbolic derivative is defined by $f^{\prime \prime}(z)=\frac{\left|f^{\prime}(z)\right|}{1-|f(z)|^{2}}$ of $f \in B(\mathbb{D})$ and the hyperbolic distance is given by $\rho(f(z), 0):=\frac{1}{2} \log \left(\frac{1+|f(z)|}{1-|f(z)|}\right)$ between $f(z)$ and zero.

A function $f \in B(\mathbb{D})$ is said to belong to the hyperbolic $\alpha$-Bloch class $\mathcal{B}_{\alpha}^{*}$ if

$$
\|f\|_{\mathcal{B}_{\alpha}^{*}}=\sup _{z \in \mathbb{D}} f^{*}(z)\left(1-|z|^{2}\right)^{\alpha}<\infty
$$

The little hyperbolic Bloch-type class $\mathcal{B}_{\alpha, 0}^{*}$ consists of all $f \in \mathcal{B}_{\alpha}^{*}$ such that

$$
\lim _{|z| \rightarrow 1} f^{\prime \prime}(z)\left(1-|z|^{2}\right)^{\alpha}=0 .
$$

The Schwarz-Pick lemma implies $\mathcal{B}_{\alpha}^{*}=B(\mathbb{D})$ for all $\alpha \geq 1$ with $\|f\|_{\mathcal{B}_{\alpha}^{\circ}} \leq 1$, and therefore, the hyperbolic $\alpha$-Bloch classes are of interest only when $0<\alpha<1$.

It is obvious that $\mathcal{B}_{\alpha}^{*}$ is not a linear space since the sum of two functions in $B(\mathbb{D})$ does not necessarily belong to $B(\mathbb{D})$.

Now, let $0<p<\infty$, we define the hyperbolic derivative by $f_{p}^{\prime \prime}(z)=\frac{p}{2} \frac{|f(z)|^{\frac{p}{2}-1}||^{\prime}(z) \mid}{1-|f(z)|^{p}}$ of $f \in$ $B(\mathbb{D})$. When $p=2$, we obtain the usual hyperbolic derivative as defined above.

A function $f \in B(\mathbb{D})$ is said to belong to the generalized hyperbolic $(p, \alpha)$-Bloch class $\mathcal{B}_{p, \alpha}^{*}$ if

$$
\|f\|_{\mathcal{B}_{p, \alpha}^{*}}=\sup _{z \in \mathbb{D}} f_{p}^{*}(z)\left(1-|z|^{2}\right)^{\alpha}<\infty .
$$

The little generalized $(p, \alpha)$-hyperbolic Bloch-type class $\mathcal{B}_{p, \alpha, 0}^{*}$ consists of all $f \in \mathcal{B}_{p, \alpha}^{*}$ such that

$$
\lim _{|z| \rightarrow 1} f_{p}^{*}(z)\left(1-|z|^{2}\right)^{\alpha}=0
$$

Let the Green's function of $\mathbb{D}$ be defined as $g(z, a)=\log \frac{1}{\left|\varphi_{a}(z)\right|}$, where $\varphi_{a}(z)=\frac{a-z}{1-\bar{a} z}$ is the Möbius transformation related to the point $a \in \mathbb{D}$. For $0<p, s<\infty$, the hyperbolic class $Q "(p, s)$ consists of those functions $f \in B(\mathbb{D})$ for which

$$
\|f\|_{Q^{*}(p, s)}^{p}=\sup _{a \in \mathbb{D}} \int_{\mathbb{D}}\left(f_{p}^{*}(z)\right)^{2} g^{s}(z, a) d A(z)<\infty .
$$

Moreover, we say that $f \in Q^{*}(p, s)$ belongs to the class $Q^{*}(p, s, 0)$ if

$$
\lim _{|a| \rightarrow 1} \int_{\mathbb{D}}\left(f_{p}^{\prime \prime}(z)\right)^{2} g^{s}(z, a) d A(z)=0 .
$$

When $p=2$, we obtain the usual hyperbolic $Q$ class as studied in $[10,11,14]$. 
Remark 1.3 The Schwarz-Pick lemma implies that $\mathcal{B}_{p, \alpha}^{*}=B(\mathbb{D})$ for all $\alpha \geq 1$ with

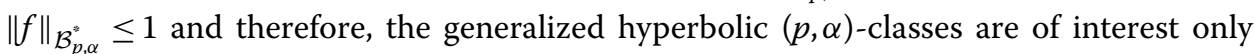
when $0<\alpha<1$. Also $Q^{\prime \prime}(p, s)=B(\mathbb{D})$ for all $s>1$, and hence, the generalized hyperbolic $Q(p, s)$-classes will be considered when $0 \leq s \leq 1$.

For any holomorphic self-mapping $\phi$ of $\mathbb{D}$, the symbol $\phi$ induces a linear composition operator $C_{\phi}(f)=f \circ \phi$ from $\mathcal{H}(\mathbb{D})$ or $B(\mathbb{D})$ into itself. The study of a composition operator $C_{\phi}$ acting on the spaces of analytic functions has engaged many analysts for many years (see, e.g., $[1-8,11,13,16]$ and others).

Yamashita was probably the first to consider systematically hyperbolic function classes. He introduced and studied hyperbolic Hardy, BMOA and Dirichlet classes in [18-20] and others. More recently, Smith studied inner functions in the hyperbolic little Bloch-class [15], and the hyperbolic counterparts of the $Q_{p}$ spaces were studied by Li in [10] and Li et al. in [11]. Further, hyperbolic $Q_{p}$ classes and composition operators were studied by Pérez-González et al. in [14].

In this paper, we will study the generalized hyperbolic $(p, \alpha)$-Bloch classes $\mathcal{B}_{p, \alpha}^{*}$ and the hyperbolic $Q^{*}(p, s)$ type classes. We will also give some results to characterize Lipschitz continuous and compact composition operators mapping from the generalized hyperbolic $(p, \alpha)$-Bloch class $\mathcal{B}_{p, \alpha}^{*}$ to $Q^{*}(p, s)$ classes by conditions depending on the symbol $\phi$ only. Thus, the results are generalizations of the recent results of Pérez-González, Rättyä and Taskinen [14].

Recall that a linear operator $T: X \rightarrow Y$ is said to be bounded if there exists a constant $C>0$ such that $\|T(f)\|_{Y} \leq C\|f\|_{X}$ for all maps $f \in X$. By elementary functional analysis, a linear operator between normed spaces is bounded if and only if it is continuous, and the boundedness is trivially also equivalent to the Lipschitz-continuity. Moreover, $T: X \rightarrow Y$ is said to be compact if it takes bounded sets in $X$ to sets in $Y$ which have compact closure. For Banach spaces $X$ and $Y$ contained in $B(\mathbb{D})$ or $\mathcal{H}(\mathbb{D}), T: X \rightarrow Y$ is compact if and only if for each bounded sequence $\left(x_{n}\right) \in X$, the sequence $\left(T x_{n}\right) \in Y$ contains a subsequence converging to a function $f \in Y$.

Throughout this paper, $C$ stands for absolute constants which may indicate different constants from one occurrence to the next.

The following lemma follows by standard arguments similar to those outlined in [17]. Hence we omit the proof.

Lemma 1.1 Assume $\phi$ is a holomorphic mapping from $\mathbb{D}$ into itself. Let $0<p, s<\infty$, and $0<\alpha<\infty$. Then $C_{\phi}: \mathcal{B}_{p, \alpha}^{*} \rightarrow Q^{\prime \prime}(p, s)$ is compact if and only if for any bounded sequence $\left(f_{n}\right)_{n \in \mathbb{N}} \in \mathcal{B}_{p, \alpha}^{*}$ which converges to zero uniformly on compact subsets of $\mathbb{D}$ as $n \rightarrow \infty$, we have $\lim _{n \rightarrow \infty}\left\|C_{\phi} f_{n}\right\|_{Q^{*}(p, s)}=0$.

Using the standard arguments similar to those outlined in Lemma 1 of [9], we have the following lemma:

Lemma 1.2 Let $0<\alpha<\infty$, then there exist two functions $f, g \in \mathcal{B}_{p, \alpha}^{*}$ such that for some constant $C$,

$$
\left(\left|f_{p}^{*}(z)\right|+\left|g_{p}^{*}(z)\right|\right)\left(1-|z|^{2}\right)^{\alpha} \geq C>0, \quad \text { for each } z \in \mathbb{D} .
$$




\section{Natural metrics in $\mathcal{B}_{p, \alpha}^{*}$ and $Q^{*}(p, s)$ classes}

In this section we introduce natural metrics on generalized hyperbolic $\alpha$-Bloch classes $\mathcal{B}_{p, \alpha}^{*}$ and the classes $Q^{*}(p, s)$.

Let $0<p, s<\infty$, and $0<\alpha<1$. First, we can find a natural metric in $\mathcal{B}_{p, \alpha}^{*}$ (see [14]) by defining

$$
d\left(f, g ; \mathcal{B}_{p, \alpha}^{*}\right):=d_{\mathcal{B}_{p, \alpha}^{*}}(f, g)+\|f-g\|_{\mathcal{B}_{p, \alpha}}+|f(0)-g(0)|^{\frac{p}{2}},
$$

where

$$
d_{\mathcal{B}_{p, \alpha}^{\circ}}(f, g):=\sup _{z \in \mathbb{D}}\left|\frac{f^{\prime}(z)|f(z)|^{\frac{p}{2}-1}}{1-|f(z)|^{p}}-\frac{g^{\prime}(z)|g(z)|^{\frac{p}{2}-1}}{1-|g(z)|^{p}}\right|\left(1-|z|^{2}\right)^{\alpha} .
$$

For $f, g \in Q^{*}(p, s)$, define their distance by

$$
d\left(f, g ; Q^{*}(p, s)\right):=d_{Q^{*}}(f, g)+\|f-g\|_{Q(p, s)}+|f(0)-g(0)|^{\frac{p}{2}},
$$

where

$$
d_{Q^{a}}(f, g):=\left(\frac{p}{2} \sup _{z \in \mathbb{D}} \int_{\mathbb{D}}\left|\frac{f^{\prime}(z)|f(z)|^{\frac{p}{2}-1}}{1-|f(z)|^{p}}-\frac{g^{\prime}(z)|g(z)|^{\frac{p}{2}-1}}{1-|g(z)|^{p}}\right|^{2} g^{s}(z, a) d A(z)\right)^{\frac{1}{2}} .
$$

Now, we give a characterization of the complete metric space $d\left(\cdot, \cdot ; \mathcal{B}_{p, \alpha}^{*}\right)$.

Proposition 2.1 The class $\mathcal{B}_{p, \alpha}^{*}$ equipped with the metric $d\left(\cdot, \cdot ; \mathcal{B}_{p, \alpha}^{*}\right)$ is a complete metric space. Moreover, $\mathcal{B}_{p, \alpha, 0}^{*}$ is a closed (and therefore complete) subspace of $\mathcal{B}_{p, \alpha}^{*}$.

Proof Clearly $d\left(f, g ; \mathcal{B}_{p, \alpha}^{*}\right) \geq 0, d\left(f, g, \mathcal{B}_{p, \alpha}^{*}\right)=d\left(g, f ; \mathcal{B}_{p, \alpha}^{*}\right)$. Also,

$$
d\left(f, h ; \mathcal{B}_{p, \alpha}^{*}\right) \leq d\left(f, g ; \mathcal{B}_{p, \alpha}^{*}\right)+d\left(g, h ; \mathcal{B}_{p, \alpha}^{*}\right)
$$

Moreover, $d\left(f, f ; \mathcal{B}_{p, \alpha}^{*}\right)=0$ for all $f, g, h \in \mathcal{B}_{p, \alpha}^{*}$.

It follows from the presence of the usual $(p, \alpha)$-Bloch term that $d\left(f, g ; \mathcal{B}_{p, \alpha}^{*}\right)=0$ implies $f=g$. Hence, $\left(\mathcal{B}_{p, \alpha}^{*}, d\right)$ is a metric space. Let $\left(f_{n}\right)_{n=1}^{\infty}$ be a Cauchy sequence in the metric space $\left(\mathcal{B}_{p, \alpha}^{*}, d\right)$, that is, for any $\varepsilon>0$, there is an $N=N(\varepsilon) \in \mathbb{N}$ such that

$$
d\left(f_{n}, f_{m} ; \mathcal{B}_{p, \alpha}^{\prime \prime}\right)<\varepsilon
$$

for all $n, m>N$. Since $\left(f_{n}\right) \subset B(\mathbb{D})$, the family $\left(f_{n}\right)$ is uniformly bounded and hence normal in $\mathbb{D}$. Therefore, there exist $f \in B(\mathbb{D})$ and a subsequence $\left(f_{n_{j}}\right)_{j=1}^{\infty}$ such that $f_{n_{j}}$ converges to $f$ uniformly on compact subsets, and by the Cauchy formula, the same also holds for the derivatives. Let $m>N$. Then the uniform convergence yields

$$
\begin{aligned}
& \left|\frac{f^{\prime}(z)|f(z)|^{\frac{p}{2}-1}}{1-|f(z)|^{p}}-\frac{f_{m}^{\prime}(z)\left|f_{m}(z)\right|^{\frac{p}{2}-1}}{1-\left|f_{m}(z)\right|^{p}}\right|\left(1-|z|^{2}\right)^{\alpha} \\
& \quad=\lim _{n \rightarrow \infty}\left|\frac{f_{n}^{\prime}(z)\left|f_{n}(z)\right|^{\frac{p}{2}-1}}{1-\left|f_{n}(z)\right|^{p}}-\frac{f_{m}^{\prime}(z)\left|f_{m}(z)\right|^{\frac{p}{2}-1}}{1-\left|f_{m}(z)\right|^{p}}\right|\left(1-|z|^{2}\right)^{\alpha} \leq \lim _{n \rightarrow \infty} d\left(f_{n}, f_{m} ; \mathcal{B}_{p, \alpha}^{*}\right) \leq \varepsilon
\end{aligned}
$$


for all $z \in \mathbb{D}$, and it follows that

$$
\|f\|_{\mathcal{B}_{p, \alpha}^{*}} \leq\left\|f_{m}\right\|_{\mathcal{B}_{p, \alpha}^{*}}+\varepsilon
$$

Thus, $f \in \mathcal{B}_{p, \alpha}^{*}$ as desired. Moreover, (2) and the completeness of the usual $(p, \alpha)$-Bloch imply that $\left(f_{n}\right)_{n=1}^{\infty}$ converges to $f$ with respect to the metric $d$. The second part of the assertion follows by (2).

Next, we give a characterization of the complete metric space $d\left(\cdot, \cdot ; Q^{*}(p, s)\right)$.

Proposition 2.2 The class $Q^{*}(p, s)$ equipped with the metric $d\left(\cdot, \cdot ; Q^{*}(p, s)\right)$ is a complete metric space. Moreover, $Q^{*}(p, s, 0)$ is a closed (and therefore complete) subspace of $Q^{*}(p, s)$.

Proof For $f, g, h \in Q^{*}(p, s)$, then clearly

- $d\left(f, g ; Q^{*}(p, s)\right) \geq 0$,

- $d\left(f, f ; Q^{*}(p, s)\right)=0$,

- $d\left(f, g ; Q^{\prime \prime}(p, s)\right)=0$ implies $f=g$,

- $d\left(f, g ; Q^{*}(p, s)\right)=d\left(g, f ; Q^{*}(p, s)\right)$,

- $d\left(f, h ; Q^{\prime \prime}(p, s)\right) \leq d\left(f, g ; Q^{\prime \prime}(p, s)\right)+d\left(g, h ; Q^{*}(p, s)\right)$.

Hence, $d$ is metric on $Q^{*}(p, s)$.

For the completeness proof, let $\left(f_{n}\right)_{n=1}^{\infty}$ be a Cauchy sequence in the metric space $\left(Q^{*}(p, s), d\right)$, that is, for any $\varepsilon>0$ there is an $N=N(\varepsilon) \in \mathbb{N}$ such that $d\left(f_{n}, f_{m} ; Q^{*}(p, s)\right)<\varepsilon$, for all $n, m>N$. Since $f_{n} \in B(\mathbb{D})$ such that $f_{n}$ converges to $f$ uniformly on compact subsets of $\mathbb{D}$. Let $m>N$ and $0<r<1$. Then Fatou's lemma yields

$$
\begin{aligned}
& \int_{D(0, r)}\left|\frac{|f(z)|^{\frac{p}{2}-1} f^{\prime}(z)}{1-|f(z)|^{p}}-\frac{\left|f_{m}(z)\right|^{\frac{p}{2}-1} f_{m}^{\prime}(z)}{1-\left|f_{m}(z)\right|^{p}}\right|^{2} g^{s}(z, a) d A(z) \\
& \quad=\int_{D(0, r)} \lim _{n \rightarrow \infty}\left|\frac{\left|f_{n}(z)\right|^{\frac{p}{2}-1} f_{n}^{\prime}(z)}{1-\left|f_{n}(z)\right|^{p}}-\frac{\left|f_{m}(z)\right|^{\frac{p}{2}-1} f_{m}^{\prime}(z)}{1-\left|f_{m}(z)\right|^{p}}\right|^{2} g^{s}(z, a) d A(z) \\
& \quad \leq \lim _{n \rightarrow \infty} \int_{\mathbb{D}}\left|\frac{\left|f_{n}(z)\right|^{\frac{p}{2}-1} f_{n}^{\prime}(z)}{1-\left|f_{n}(z)\right|^{p}}-\frac{\left|f_{m}(z)\right|^{\frac{p}{2}-1} f_{m}^{\prime}(z)}{1-\left|f_{m}(z)\right|^{p}}\right|^{2} g^{S}(z, a) d A(z) \leq \varepsilon^{2},
\end{aligned}
$$

and by letting $r \rightarrow 1^{-}$, it follows that

$$
\int_{\mathbb{D}}\left(f_{p}^{\prime \prime}(z)\right)^{2} g^{s}(z, a) d A(z) \leq 2 \varepsilon^{2}+2 \int_{\mathbb{D}}\left|\frac{\left|f_{m}(z)\right|^{\frac{p}{2}-1} f_{m}^{\prime}(z)}{1-\left|f_{m}(z)\right|^{p}}\right|^{2} g^{s}(z, a) d A(z) .
$$

This yields

$$
\|f\|_{Q^{\prime \prime}(p, s)}^{p} \leq 2 \varepsilon^{2}+2\left\|f_{m}\right\|_{Q^{\prime \prime}(p, s)}^{2},
$$

and thus $f \in Q^{\prime \prime}(p, s)$. We also find that $f_{n} \rightarrow f$ with respect to the metric of $Q^{*}(p, s)$. The second part of the assertion follows by (3).

\section{Lipschitz continuous and compactness of $C_{\phi}$}

Theorem 3.1 Let $0<p<\infty, 0 \leq s \leq 1$, and $0<\alpha \leq 1$. Assume that $\phi$ is a holomorphic mapping from $\mathbb{D}$ into itself. Then the following statements are equivalent: 
(i) $C_{\phi}: \mathcal{B}_{p, \alpha}^{*} \rightarrow Q^{*}(p, s)$ is bounded;

(ii) $C_{\phi}: \mathcal{B}_{p, \alpha}^{\prime \prime} \rightarrow Q^{\prime \prime}(p, s)$ is Lipschitz continuous;

(iii) $\sup _{a \in \mathbb{D}} \int_{\mathbb{D}} \frac{\left|\phi^{\prime}(z)\right|^{2}}{\left(1-|\phi(z)|^{p}\right)^{2 \alpha}} g^{s}(z, a) d A(z)<\infty$.

Proof First, assume that (i) holds, then there exists a constant $C$ such that

$$
\left\|C_{\phi} f\right\|_{Q^{*}(p, s)} \leq C\|f\|_{\mathcal{B}_{p, \alpha}^{*}}, \quad \text { for all } f \in \mathcal{B}_{p, \alpha}^{*}
$$

For given $f \in \mathcal{B}_{p, \alpha}^{*}$, the function $f_{t}(z)=f(t z)$, where $0<t<1$, belongs to $\mathcal{B}_{p, \alpha}^{*}$ with the property $\left\|f_{t}\right\|_{\mathcal{B}_{p, \alpha}^{*}} \leq\|f\|_{\mathcal{B}_{p, \alpha}^{*}}$. Let $f, g$ be the functions from Lemma 1.2 such that

$$
\frac{1}{\left(1-|z|^{2}\right)^{\alpha}} \leq\left|f_{p}^{\prime \prime}(z)\right|+\left|g_{p}^{*}(z)\right|
$$

for all $z \in \mathbb{D}$, so that

$$
\frac{\left|\phi^{\prime}(z)\right|}{(1-|\phi(z)|)^{\alpha}} \leq(f \circ \phi)^{*}(z)+(g \circ \phi)^{\prime \prime}(z) .
$$

Thus,

$$
\begin{aligned}
& \int_{\mathbb{D}} \frac{\left|t \phi^{\prime}(z)\right|^{2}}{\left(1-|t \phi(z)|^{2}\right)^{2 \alpha}} g^{s}(z, a) d A(z) \\
& \quad \leq C \int_{\mathbb{D}}\left(\left((f \circ t \phi)_{p}^{*}(z)\right)^{2}+\left((g \circ t \phi)_{p}^{*}(z)\right)^{2}\right) g^{s}(z, a) d A(z) \\
& \quad \leq C\left\|C_{\phi}\right\|^{2}\left(\|f\|_{\mathcal{B}_{p, \alpha}^{*}}^{2}+\|g\|_{\mathcal{B}_{p, \alpha}^{*}}^{2}\right) .
\end{aligned}
$$

This estimate together with the Fatou's lemma implies (iii).

Conversely, assuming that (iii) holds and that $f \in \mathcal{B}_{p, \alpha}^{*}$, we see that

$$
\begin{aligned}
& \sup _{a \in \mathbb{D}} \int_{\mathbb{D}}\left((f \circ \phi)_{p}^{*}(z)\right)^{2} g^{s}(z, a) d A(z) \\
& \quad=\sup _{a \in \mathbb{D}} \int_{\mathbb{D}}\left(f_{p}^{*}(\phi(z))\right)^{2}\left|\phi^{\prime}(z)\right|^{2} g^{s}(z, a) d A(z) \\
& \leq\|f\|_{\mathcal{B}_{p, \alpha}^{*}}^{2} \sup _{a \in \mathbb{D}} \int_{\mathbb{D}} \frac{\left|\phi^{\prime}(z)\right|^{2}}{\left(1-|\phi(z)|^{p}\right)^{2 \alpha}} g^{s}(z, a) d A(z) .
\end{aligned}
$$

Hence, it follows that (i) holds.

(ii) $\Longleftrightarrow$ (iii). Assume first that $C_{\phi}: \mathcal{B}_{p, \alpha}^{*} \rightarrow Q^{*}(p, s)$ is Lipschitz continuous, that is, there exists a positive constant $C$ such that

$$
d\left(f \circ \phi, g \circ \phi ; Q^{*}(p, s)\right) \leq C d\left(f, g ; \mathcal{B}_{p, \alpha}^{*}\right), \quad \text { for all } f, g \in \mathcal{B}_{p, \alpha}^{*} .
$$

Taking $g=0$, this implies

$$
\|f \circ \phi\|_{Q^{*}(p, s)} \leq C\left(\|f\|_{\mathcal{B}_{p, \alpha}^{*}}+\|f\|_{\mathcal{B}_{p, \alpha}}+|f(0)|^{\frac{p}{2}}\right), \quad \text { for all } f \in \mathcal{B}_{p, \alpha}^{*} .
$$


The assertion (iii) for $\alpha=1$ follows by choosing $f(z)=z$ in (4). If $0<\alpha<1$, then

$$
\begin{aligned}
\frac{2}{p}|f(z)|^{\frac{p}{2}} & =\left.\left|\int_{0}^{z}\right| f(t)\right|^{\frac{p}{2}} f^{\prime}(t) d t+(f(0))^{\frac{p}{2}} \mid \\
& \leq\|f\|_{\mathcal{B}_{p, \alpha}} \int_{0}^{|z|} \frac{d x}{\left(1-x^{2}\right)^{\alpha}}+|f(0)|^{\frac{p}{2}} \\
& \leq \frac{\|f\|_{\mathcal{B}_{\alpha}}}{(1-\alpha)}+|f(0)|^{\frac{p}{2}}
\end{aligned}
$$

this yields

$$
\frac{2}{p}|f(\phi(0))-g(\phi(0))|^{\frac{p}{2}} \leq \frac{\|f-g\|_{\mathcal{B}_{p, \alpha}}}{(1-\alpha)}+|f(0)-g(0)|^{\frac{p}{2}}
$$

Moreover, Lemma 1.2 implies the existence of $f, g \in \mathcal{B}_{p, \alpha}^{*}$ such that

$$
\left|f_{p}^{*}(z)+g_{p}^{*}(z)\right|\left(1-|z|^{2}\right)^{\alpha} \geq C>0, \quad \text { for all } z \in \mathbb{D} \text {. }
$$

Combining (4) and (5), we obtain

$$
\begin{aligned}
& \|f\|_{\mathcal{B}_{p, \alpha}^{*}}+\|g\|_{\mathcal{B}_{p, \alpha}^{*}}+\|f\|_{\mathcal{B}_{p, \alpha}}+\|g\|_{\mathcal{B}_{p, \alpha}}+|f(0)|^{\frac{p}{2}}+|g(0)|^{\frac{p}{2}} \\
& \quad \geq C \int_{\mathbb{D}} \frac{\left|\phi^{\prime}(z)\right|^{2}}{\left(1-|\phi(z)|^{p}\right)^{2 \alpha}} g^{s}(z, a) d A(z)
\end{aligned}
$$

for which the assertion (iii) follows.

Assume now that (iii) is satisfied, we have

$$
\begin{aligned}
d\left(f \circ \phi, g \circ \phi ; Q^{*}(p, s)\right)= & d_{Q_{(p, s)}^{*}}(f \circ \phi, g \circ \phi)+\|f \circ \phi-g \circ \phi\|_{Q(p, s)} \\
& +|f(\phi(0))-g(\phi(0))|^{\frac{p}{2}} \\
\leq & d_{\mathcal{B}_{p, \alpha}^{*}}(f, g)\left(\sup _{a \in \mathbb{D}} \int_{\mathbb{D}} \frac{\left|\phi^{\prime}(z)\right|^{2}}{\left(1-|\phi(z)|^{p}\right)^{2 \alpha}} g^{s}(z, a) d A(z)\right)^{\frac{1}{2}} \\
& +\|f-g\|_{\mathcal{B}_{p, \alpha}}\left(\sup _{a \in \mathbb{D}} \int_{\mathbb{D}} \frac{\left|\phi^{\prime}(z)\right|^{2}}{\left(1-|\phi(z)|^{p}\right)^{2 \alpha}} g^{s}(z, a) d A(z)\right)^{\frac{1}{2}} \\
& +\frac{\|f-g\|_{\mathcal{B}_{p, \alpha}}}{(1-\alpha)}+|f(0)-g(0)|^{\frac{p}{2}} \\
\leq & C d\left(f, g ; \mathcal{B}_{p, \alpha}^{*}\right) .
\end{aligned}
$$

Thus $C_{\phi}: \mathcal{B}_{p, \alpha}^{*} \rightarrow Q^{*}(p, s)$ is Lipschitz continuous and the proof is completed.

Remark 3.1 We know that a composition operator $C_{\phi}: \mathcal{B}_{p, \alpha}^{*} \rightarrow Q^{*}(p, s)$ is said to be bounded if there is a positive constant $C$ such that $\left\|C_{\phi} f\right\|_{Q^{*}(p, s)} \leq C\|f\|_{\mathcal{B}_{p, \alpha}^{*}}$ for all $f \in \mathcal{B}_{p, \alpha}^{*}$. Theorem 3.1 shows that $C_{\phi}: \mathcal{B}_{p, \alpha}^{*} \rightarrow Q^{*}(p, s)$ is bounded if and only if it is Lipschitzcontinuous, that is, if there exists a positive constant $C$ such that

$$
d\left(f \circ \phi, g \circ \phi ; Q^{*}(p, s)\right) \leq C d\left(f, g ; \mathcal{B}_{p, \alpha}^{*}\right), \quad \text { for all } f, g \in \mathcal{B}_{p, \alpha}^{*} .
$$


By elementary functional analysis, a linear operator between normed spaces is bounded if and only if it is continuous, and the boundedness is trivially also equivalent to the Lipschitz-continuity. So, our result for composition operators in hyperbolic spaces is the correct and natural generalization of the linear operator theory.

Recall that a composition operator $C_{\phi}: \mathcal{B}_{p, \alpha}^{*} \rightarrow Q^{*}(p, s)$ is compact if it maps any ball in $\mathcal{B}_{p, \alpha}^{*}$ onto a precompact set in $Q^{*}(p, s)$.

The following observation is sometimes useful.

Proposition 3.1 Let $0<p<\infty, 0 \leq s \leq 1$ and $0<\alpha \leq 1$. Assume that $\phi$ is a holomorphic mapping from $\mathbb{D}$ into itself. If $C_{\phi}: \mathcal{B}_{p, \alpha}^{*} \rightarrow Q^{*}(p, s)$ is compact, it maps closed balls onto compact sets.

Proof If $B \subset \mathcal{B}_{p, \alpha}^{*}$ is a closed ball and $g \in Q^{*}(p, s)$ belongs to the closure of $C_{\phi}(B)$, we can find a sequence $\left(f_{n}\right)_{n=1}^{\infty} \subset B$ such that $f_{n} \circ \phi$ converges to $g \in Q^{*}(p, s)$ as $n \rightarrow \infty$. But $\left(f_{n}\right)_{n=1}^{\infty}$ is a normal family, hence it has a subsequence $\left(f_{n_{j}}\right)_{j=1}^{\infty}$ converging uniformly on the compact subsets of $\mathbb{D}$ to an analytic function $f$. As in earlier arguments of Proposition 2.1 in [14], we get a positive estimate which shows that $f$ must belong to the closed ball $B$. On the other hand, also the sequence $\left(f_{n_{j}} \circ \phi\right)_{j=1}^{\infty}$ converges uniformly on compact subsets to an analytic function, which is $g \in Q^{*}(p, s)$. We get $g=f \circ \phi$, i.e., $g$ belongs to $C_{\phi}(B)$. Thus, this set is closed and also compact.

Compactness of composition operators can be characterized in full analogy with the linear case.

Theorem 3.2 Let $0<p<\infty, 0 \leq s \leq 1$, and $0<\alpha \leq 1$. Assume that $\phi$ is a holomorphic mapping from $\mathbb{D}$ into itself. Then the following statements are equivalent:

(i) $C_{\phi}: \mathcal{B}_{p, \alpha}^{*} \rightarrow Q^{*}(p, s)$ is compact.

(ii)

$$
\lim _{r \rightarrow 1^{-}} \sup _{a \in \mathbb{D}} \int_{|\phi| \geq r_{j}} \frac{\left|\phi^{\prime}(z)\right|^{2}}{\left(1-|\phi(z)|^{p}\right)^{2 \alpha}} g^{s}(z, a) d A(z)=0 .
$$

Proof We first assume that (ii) holds. Let $B:=\bar{B}(g, \delta) \subset \mathcal{B}_{p, \alpha}^{*}$, where $g \in \mathcal{B}_{p, \alpha}^{*}$ and $\delta>0$, be a closed ball, and let $\left(f_{n}\right)_{n=1}^{\infty} \subset B$ be any sequence. We show that its image has a convergent subsequence in $Q^{*}(p, s)$, which proves the compactness of $C_{\phi}$ by definition.

Again, $\left(f_{n}\right)_{n=1}^{\infty} \subset B(\mathbb{D})$ is a normal family, hence there is a subsequence $\left(f_{n_{j}}\right)_{j=1}^{\infty}$ which converges uniformly on the compact subsets of $\mathbb{D}$ to an analytic function $f$. By the Cauchy formula for the derivative of an analytic function, also the sequence $\left(f_{n_{j}}^{\prime}\right)_{j=1}^{\infty}$ converges uniformly to $f^{\prime}$. It follows that also the sequences $\left(f_{n_{j}} \circ \phi\right)_{j=1}^{\infty}$ and $\left(f_{n_{j}}^{\prime} \circ \phi\right)_{j=1}^{\infty}$ converge uniformly on the compact subsets of $\mathbb{D}$ to $f \circ \phi$ and $f^{\prime} \circ \phi$, respectively. Moreover, $f \in B \subset \mathcal{B}_{p, \alpha}^{*}$ since for any fixed $R, 0<R<1$, the uniform convergence yields

$$
\begin{aligned}
\sup _{|z| \leq R} \mid & \left|\frac{f^{\prime}(z)|f(z)|^{\frac{p}{2}-1}}{1-|f(z)|^{p}}-\frac{g^{\prime}(z)|g(z)|^{\frac{p}{2}-1}}{1-|g(z)|^{p}}\right|\left(1-|z|^{2}\right)^{\alpha} \\
& +\sup _{|z| \leq R}|f(z)-g(z)|^{\frac{p}{2}-1}\left|f^{\prime}(z)-g^{\prime}(z)\right|\left(1-|z|^{2}\right)^{\alpha}+|f(0)-g(0)|^{\frac{p}{2}-1}
\end{aligned}
$$




$$
\begin{aligned}
= & \lim _{j \rightarrow \infty} \sup _{|z| \leq R}\left|\frac{f_{n_{j}}^{\prime}(z)\left|f_{n_{j}}(z)\right|^{\frac{p}{2}-1}}{1-\left|f_{n_{j}}(z)\right|^{p}}-\frac{g^{\prime}(z)|g(z)|^{\frac{p}{2}-1}}{1-|g(z)|^{p}}\right|\left(1-|z|^{2}\right)^{\alpha} \\
& +\sup _{|z| \leq R}\left|f_{n_{j}}(z)-g(z)\right|^{\frac{p}{2}-1}\left|f_{n_{j}}^{\prime}(z)-g^{\prime}(z)\right|\left(1-|z|^{2}\right)^{\alpha}+|f(0)-g(0)|^{\frac{p}{2}-1} \leq \delta .
\end{aligned}
$$

Hence, $d\left(f, g ; \mathcal{B}_{p, \alpha}^{*}\right) \leq \delta$.

Let $\varepsilon>0$. Since (ii) is satisfied, we may fix $r, 0<r<1$, such that

$$
\sup _{a \in \mathbb{D}} \int_{|\phi(z)| \geq r} \frac{|\phi(z)|^{p-2}\left|\phi^{\prime}(z)\right|^{2}}{\left(1-|\phi(z)|^{p}\right)^{2 \alpha}} g^{s}(z, a) d A(z) \leq \varepsilon
$$

By the uniform convergence, we may fix $N_{1} \in \mathbb{N}$ such that

$$
\left|f_{n_{j}} \circ \phi(0)-f \circ \phi(0)\right| \leq \varepsilon, \quad \text { for all } j \geq N_{1} .
$$

The condition (ii) is known to imply the compactness of $C_{\phi}: \mathcal{B}_{p, \alpha} \rightarrow Q(p, s)$, hence possibly to passing once more to a subsequence and adjusting the notations, we may assume that

$$
\left\|f_{h_{j}} \circ \phi-f \circ \phi\right\|_{Q(p, s)} \leq \varepsilon, \quad \text { for all } j \geq N_{2} \text {, for some } N_{2} \in \mathbb{N} \text {. }
$$

Now let

$$
I_{1}(a, r)=\sup _{a \in \mathbb{D}} \int_{|\phi(z)| \geq r}\left[\left(f_{n_{j}} \circ \phi\right)_{p}^{*}(z)-(f \circ \phi)_{p}^{*}(z)\right]^{2} g^{s}(z, a) d A(z),
$$

and

$$
I_{2}(a, r)=\sup _{a \in \mathbb{D}} \int_{|\phi(z)| \leq r}\left[\left(f_{n_{j}} \circ \phi\right)_{p}^{*}(z)-(f \circ \phi)_{p}^{*}(z)\right]^{2} g^{s}(z, a) d A(z) .
$$

Since $\left(f_{n_{j}}\right)_{j=1}^{\infty} \subset B$ and $f \in B$, it follows that

$$
\begin{aligned}
I_{1}(a, r) & =\sup _{a \in \mathbb{D}} \int_{|\phi(z)| \geq r}\left[\left(f_{n_{j}} \circ \phi\right)_{p}^{*}(z)-(f \circ \phi)_{p}^{*}(z)\right]^{2} g^{s}(z, a) d A(z) \\
& \leq \frac{p}{2} \sup _{a \in \mathbb{D}} \int_{|\phi(z)| \geq r} \mathcal{L}\left(f_{n_{j}}, f, \phi\right) g^{s}(z, a) d A(z) \\
& \leq d_{\mathcal{B}_{\alpha}^{*}}\left(f_{n_{j}}, f\right) \sup _{a \in \mathbb{D}} \int_{|\phi(z)| \geq r} \frac{\left|\phi^{\prime}(z)\right|^{2}}{\left(1-|\phi(z)|^{p}\right)^{2 \alpha}} g^{s}(z, a) d A(z),
\end{aligned}
$$

where

$$
\mathcal{L}\left(f_{n_{j}}, f, \phi\right)=\left|\frac{\left|\left(f_{n_{j}} \circ \phi\right)(z)\right|^{\frac{p}{2}-1}\left(f_{n_{j}} \circ \phi\right)^{\prime}(z)}{1-\left|\left(f_{n_{j}} \circ \phi\right)(z)\right|^{p}}-\frac{|(f \circ \phi)(z)|^{\frac{p}{2}-1}(f \circ \phi)^{\prime}(z)}{1-|(f \circ \phi)(z)|^{p}}\right|^{2} .
$$

Hence,

$$
I_{1}(a, r) \leq C \varepsilon .
$$


On the other hand, by the uniform convergence on compact subsets of $\mathbb{D}$, we can find an $N_{3} \in \mathbb{N}$ such that for all $j \geq N_{3}$,

$$
\mathcal{L}_{1}\left(f_{n_{j}}, f, \phi\right)=\left|\frac{\left|\left(f_{n_{j}} \circ \phi\right)(z)\right|^{\frac{p}{2}-1} f_{n_{j}}^{\prime}(\phi(z))}{1-\left|f_{n_{j}}(\phi(z))\right|^{p}}-\frac{|(f \circ \phi)(z)|^{\frac{p}{2}-1} f^{\prime}(\phi(z))}{1-|f(\phi(z))|^{p}}\right| \leq \varepsilon
$$

for all $z \in \mathbb{D}$ with $|\phi(z)| \leq r$. Hence, for such $j$, we obtain

$$
\begin{aligned}
I_{2}(a, r) & =\sup _{a \in \mathbb{D}} \int_{|\phi(z)| \leq r}\left(\left(f_{n_{j}} \circ \phi\right)_{p}^{\prime \prime}(z)-(f \circ \phi)_{p}^{*}(z)\right)^{2} g^{s}(z, a) d A(z) \\
& \leq \sup _{a \in \mathbb{D}} \int_{|\phi(z)| \leq r} \mathcal{L}_{1}\left(f_{n_{j}}, f, \phi\right)\left|\phi^{\prime}(z)\right|^{2} g^{s}(z, a) d A(z) \\
& \leq \varepsilon\left(\sup _{a \in \mathbb{D}} \int_{|\phi(z)| \leq r} \frac{\left|\phi^{\prime}(z)\right|^{2}}{\left(1-|\phi(z)|^{p}\right)^{2 \alpha}} g^{s}(z, a) d A(z)\right)^{\frac{1}{2}} \leq C \varepsilon,
\end{aligned}
$$

hence,

$$
I_{2}(a, r) \leq C \varepsilon
$$

where $C$ is the bound obtained from (iii) of Theorem 3.1. Combining (6), (7), (8) and (9), we deduce that $f_{n_{j}} \rightarrow f$ in $Q^{*}(p, s)$.

As for the converse direction, let $f_{n}(z):=\frac{1}{2} n^{\alpha-1} z^{n}$ for all $n \in \mathbb{N}, n \geq 2$.

$$
\begin{aligned}
\|f\|_{\mathcal{B}_{p, \alpha}^{*}} & =\frac{p}{2} \sup _{a \in \mathbb{D}} \frac{n^{\frac{\alpha p}{2}}|z|^{\frac{\alpha p}{2}}-1}{1-\left.2^{-p} n^{p(\alpha-1)}|z|^{2}\right|^{\alpha p}} \\
& \leq\left(2^{p-1}+1\right) \sup _{a \in \mathbb{D}} n^{\frac{\alpha p}{2}}|z|^{\frac{\alpha p}{2}-1}\left(1-|z|^{2}\right)^{\alpha} .
\end{aligned}
$$

The function $r^{\frac{n p}{2}-1}(1-r)^{\alpha}$ attains its maximum at the point $r=1-\frac{\alpha}{\alpha+\frac{\alpha p}{2}-1}$. For simplicity, we see that (10) has the upper bound

$$
\left(2^{p-1}+1\right) n^{\alpha}\left(1-\frac{\alpha}{\alpha+n-1}\right)^{n-1}\left(\frac{\alpha}{\alpha+n-1}\right)^{\alpha} \leq\left(2^{p-1}+1\right) .
$$

Then the sequence $\left(f_{n}\right)_{n=1}^{\infty}$ belongs to the ball $\bar{B}\left(0,\left(2^{p-1}+1\right)\right) \subset \mathcal{B}_{p, \alpha}^{*}$.

Suppose that $C_{\phi}$ maps the closed ball $\bar{B}\left(0,\left(2^{p-1}+1\right)\right) \subset \mathcal{B}_{p, \alpha}^{*}$ into a compact subset of $Q^{\prime \prime}(p, s)$; hence, there exists an unbounded increasing subsequence $\left(n_{j}\right)_{j=1}^{\infty}$ such that the image subsequence $\left(C_{\phi} f_{n_{j}}\right)_{j=1}^{\infty}$ converges with respect to the norm. Since both $\left(f_{n}\right)_{n=1}^{\infty}$ and $\left(C_{\phi} f_{n_{j}}\right)_{j=1}^{\infty}$ converge to the zero function uniformly on compact subsets of $\mathbb{D}$, the limit of the latter sequence must be zero. Hence,

$$
\left\|n_{j}^{\alpha-1} \phi^{n_{j}}\right\|_{Q^{*}(p, s)} \rightarrow 0, \quad \text { as } j \rightarrow \infty .
$$

Now let $r_{j}=1-\frac{1}{n_{j}}$. For all numbers $a, r_{j} \leq a<1$, we have the estimate

$$
\frac{n_{j}^{\alpha} a^{n_{j}-1}}{1-a^{n_{j}}} \geq \frac{1}{e(1-a)^{\alpha}} \quad(\text { see }[14])
$$


Using (12), we deduce

$$
\begin{aligned}
\left\|n_{j}^{\alpha-1} \phi^{n_{j}}\right\|_{Q^{\prime}(p, s)}^{2} & \geq \frac{p}{2} \sup _{a \in \mathbb{D}} \int_{|\phi| \geq r_{j}}\left|\frac{n_{j}^{\alpha}(\phi(z))^{n_{j}-1}\left|\phi^{n_{j}}(z)\right|^{\frac{p}{2}-1} \phi^{\prime}(z)}{1-\left|\phi^{n_{j}}(z)\right|^{p}}\right|^{2} g^{s}(z, a) d A(z) \\
& \geq \frac{C p}{8 e^{2}} \sup _{a \in \mathbb{D}} \int_{|\phi| \geq r_{j}} \frac{\left|\phi^{\prime}(z)\right|^{2}}{\left(1-|\phi(z)|^{p}\right)^{2 \alpha}} g^{s}(z, a) d A(z) .
\end{aligned}
$$

From (11) and (13), the condition (ii) follows. This completes the proof.

For $0<p<\infty$ and $0 \leq s<\infty$, we define the weighted Dirichlet-class $\mathcal{D}(p, s)$ consists of those functions $f \in \mathcal{H}(\mathbb{D})$ for which

$$
\int_{\mathbb{D}}|f(z)|^{p-2}\left|f^{\prime}(z)\right|^{2}\left(1-|z|^{2}\right)^{s} d A(z)<\infty
$$

For $0<p<\infty$ and $0 \leq s<\infty$, the generalized hyperbolic weighted Dirichlet-class $\mathcal{D}^{*}(p, s)$ consists of those functions $f \in B(\mathbb{D})$ for which

$$
\int_{\mathbb{D}}\left(f_{p}^{* \prime}(z)\right)^{2}\left(1-|z|^{2}\right)^{s} d A(z)<\infty
$$

The proof of Proposition 2.2 implies the following corollary:

Corollary 3.1 For $f, g \in \mathcal{D}^{\prime \prime}(p, s)$. Then, $\mathcal{D}^{\prime \prime}(p, s)$ is a complete metric space with respect to the metric defined by

$$
d\left(f, g ; \mathcal{D}^{\prime \prime}(p, s)\right):=d_{\mathcal{D}^{*}(p, s)}(f, g)+\|f-g\|_{\mathcal{D}(p, s)}+|f(0)-g(0)|^{\frac{p}{2}},
$$

where

$$
d_{\mathcal{D}^{\prime \prime}(p, s)}(f, g):=\left(\frac{p}{2} \sup _{z \in \mathbb{D}} \int_{\mathbb{D}}\left|\frac{f^{\prime}(z)|f(z)|^{\frac{p}{2}-1}}{1-|f(z)|^{p}}-\frac{g^{\prime}(z)|g(z)|^{\frac{p}{2}-1}}{1-|g(z)|^{p}}\right|^{2}\left(1-|z|^{2}\right)^{s} d A(z)\right)^{\frac{1}{2}} .
$$

Moreover, the proofs of Theorems 3.1 and 3.2 yield the following result:

Theorem 3.3 Let $0<p<\infty,-1<s \leq 1$, and $0<\alpha \leq 1$. Assume that $\phi$ is a holomorphic mapping from $\mathbb{D}$ into itself. Then the following statements are equivalent:

(i) $C_{\phi}: \mathcal{B}_{p, \alpha}^{*} \rightarrow \mathcal{D}^{*}(p, s)$ is Lipschitz continuous;

(ii) $C_{\phi}: \mathcal{B}_{p, \alpha}^{*} \rightarrow \mathcal{D}^{*}(p, s)$ is compact;

(iii)

$$
\int_{\mathbb{D}} \frac{\left|\phi^{\prime}(z)\right|^{2}}{\left(1-|\phi(z)|^{p}\right)^{2 \alpha}}\left(1-|z|^{2}\right)^{s} d A(z)<\infty
$$




\section{References}

1. El-Sayed Ahmed, A, Bakhit, MA: Composition operators on some holomorphic Banach function spaces. Math. Scand. 104(2), 275-295 (2009)

2. El-Sayed Ahmed, A, Bakhit, MA: Composition operators acting between some weighted Möbius invariant spaces. Ann. Funct. Anal. 2(2), 138-152 (2011)

3. Aulaskari, R, Zhao, R: Composition operators and closures of some Möbius invariant spaces in the Bloch space. Math. Scand. 107(1), 139-149 (2010)

4. Cowen, C, MacCluer, BD: Composition Operators on Spaces of Analytic Functions. Studies in Advanced Mathematics. CRC Press, Boca Raton (1995)

5. Demazeux, R: Essential norms of weighted composition operators between Hardy spaces $H^{p}$ and $H^{q}$ for $1 \leq p, q \leq \infty$. Stud. Math. 206(3), 191-209 (2011)

6. El-Fallah, O, Kellay, K, Shabankhah, M, Youssfi, M: Level sets and composition operators on the Dirichlet space. J. Funct. Anal. 260(6), 1721-1733 (2011)

7. Kellay, K, Lefére, P: Compact composition operators on weighted Hilbert spaces of analytic functions. J. Math. Anal. Appl. 386(2), 718-727 (2012)

8. Kotilainen, M: Studies on composition operators and function spaces. Report Series. Dissertation, Department of Mathematics, University of Joensuu 11 (2007)

9. Lappan, P, Xiao, J: $\mathcal{Q}_{\alpha}^{\#}$-bounded composition maps on normal classes. Note Mat. 20(1), 65-72 (2000/2001)

10. Li, X: On hyperbolic Q classes. Dissertation, University of Joensuu, Joensuu. Ann. Acad. Sci. Fenn. Math. Diss. 145 (2005)

11. Li, X, Pérez-González, F, Rättyä, J: Composition operators in hyperbolic Q-classes. Ann. Acad. Sci. Fenn. Math. 31, 391-404 (2006)

12. Li, S, Stević, S: Products of integral-type operators and composition operators between Bloch-type spaces. J. Math. Anal. Appl. 349(2), 596-610 (2009)

13. Manhas, J, Zhao, R: New estimates of essential norms of weighted composition operators between Bloch type spaces. J. Math. Anal. Appl. 389(1), 32-47 (2012)

14. Pérez-González, F, Rättyä, J, Taskinen, J: Lipschitz continuous and compact composition operators in hyperbolic classes. Mediterr. J. Math. 8, 123-135 (2011)

15. Smith, W: Inner functions in the hyperbolic little Bloch class. Mich. Math. J. 45(1), 103-114 (1998)

16. Singh, RK, Manhas, JS: Composition Operators on Function Spaces. North-Holland Mathematics Studies (1993)

17. Tjani, M: Compact composition operators on Besov spaces. Trans. Am. Math. Soc. 355, 4683-4698 (2003)

18. Yamashita, S: Hyperbolic Hardy classes and hyperbolically Dirichlet-finite functions. Hokkaido Math. J. 10, 709-722 (1981)

19. Yamashita, S: Functions with $H^{P}$ hyperbolic derivative. Math. Scand. 53(2), 238-244 (1983)

20. Yamashita, S: Holomorphic functions of hyperbolic bounded mean oscillation. Boll. Unione Mat. Ital. 5(3), 983-1000 (1986)

\section{Submit your manuscript to a SpringerOpen ${ }^{\circ}$ journal and benefit from:}

- Convenient online submission

- Rigorous peer review

- Immediate publication on acceptance

- Open access: articles freely available online

- High visibility within the field

- Retaining the copyright to your article 